\title{
Repairing Ti-6Al-4V aeronautical components with DED additive manufacturing
}

\author{
Matthieu Rauch $^{1,2}$ a), Jean-Yves Hascoët ${ }^{1,2}$ b), Manjaiah Mallaiah ${ }^{3}$ c) \\ ${ }^{1}$ Centrale Nantes / GeM- UMR CNRS 6183, - 1 rue de la Noe, 44321 Nantes, France \\ 2 Joint Laboratory of Marine Technology (JLMT) Centrale Nantes - Naval Group. \\ ${ }^{3}$ Manipal Institute of Technology (MIT), MANIPAL - 576104 Udupi Dist., Karnataka, India \\ a) matthieu.rauch@ec-nantes.fr - b) ean-yves.hascoet@ec-nantes.fr - c) manjaiah.m@manipal.edu
}

\begin{abstract}
$\underline{\text { Abstract }}$
Direct Energy Deposition (DED) processes are Additive Manufacturing (AM) processes that provide new perspectives for the manufacturing industry. In particular the area of component repair could highly benefit from these processes. It is consequently necessary to ensure the ability of DED processes, so that the repaired component can provide the same level of service than a new one. This paper focuses on the repair of Ti-6Al-4V parts by powder based LMD AM and investigates its accuracy, repeatability and reliability. At first, an experimental campaign has been carried out to evaluate the characteristics of as-built material. Optimal process parameter selection is made by a porosity and macrostructure analysis. Tensile properties, Low Cycle Fatigue and crack propagation studies have been done on as-built samples (100\% AM) and interface samples (50\% AM / $50 \%$ substrate). The results compare to wrought alloy and validate the relevance of LMD to produce sound repaired parts. In a second section, the paper proposes a semi automatic repair method of Ti-6Al-4V components: the defect geometry and the CAD model of the part to repair are identified from 3D scanning operations. Adapted additive and machining tool paths are then generated on the selected equipment.
\end{abstract}

\section{Introduction}

Laser Metal Deposition (LMD) processes are a class of Additive Manufacturing (AM) processes that provide new perspectives for the manufacturing industry [1]. Hence, the area of component repair could highly benefit from these processes by dramatically increasing the life of complex components made of high value materials, like aeronautical parts for example. It is consequently necessary to ensure the ability of LMD processes to bring the added material at the right location and with the right properties, so that the repaired component can provide the same level of service than a new one. Two aspects are particularly interesting for investigation: first the 
ability to deposit with LMD a material that will meet the requirement, and second the ability to deposit the material at the right place on the component to repair.

Some researchers [2]-[6] have studied the microstructural and mechanical properties of Ti64 alloy using additive manufacturing (AM). They found that the fabricated samples have columnar microstructure prior beta grains which are grown epitaxial from the substrate material towards the build direction. Besides these, few authors [7], [8] studied the relationship between the AM process, microstructure and resulting properties of LMD Ti64 alloys, and they provided a key recommendation for understanding LMD of Ti64 alloys. Though the microstructure of wrought alloy and LMD process are different, the tensile properties are comparable after the heat treatment. Within the context of repair, the performances at the interfaces between repaired and original alloy have been little studied.

Concerning the second aspect, about the automatization repair process, there have been several studies about LMD process parametrization and the metallurgical properties of the repaired area [9], [10] but fewer studies have focused on the automation of the LMD repair process [11], [12] and most of them based on 3D scan and $\mathrm{CAD}$ model comparison. These methods typically require significant human intervention to preprocess the range data obtained by the 3D scanner and reconstruct the geometry of the repair volume. To circumvent these limitations, the research group has been working on a semi-automatic repair method [13].

Within this context, this paper focuses on the repair of Ti-6Al-4V parts by powder based LMD AM. At first, an experimental campaign has been carried out to evaluate the characteristics of as-built material. Optimal process parameter selection is made by a porosity and macrostructure analysis. Tensile properties, Low Cycle Fatigue and crack propagation studies are done on as-built samples (100\% AM) and interface samples (50\% AM / 50\% substrate). In a second section, the paper proposes the use of a semi-automatic repair method of Ti-6Al-4V components: the defect geometry and the CAD model of the part to repair are identified from 3D scanning operations. Adapted additive and machining tool paths are then generated on the selected equipment.

\section{Mechanical properties of Ti-6Al-4V components obtained by LMD}

\section{Manufacturing of the LMD samples}

The selected TI-6Al-4V powder was Argon gas atomized and prepared to have spherical particle size of 40$90 \mu \mathrm{m}$, with the mean particle size diameter of $60 \mu \mathrm{m}$. The powder properties were analyzed using the SEM to study morphology and chemical composition (Figure 1). The particles are generally spherical with minor satellite present on the surfaces. There is no visible pore or agglomeration observed in the surface, which is a good indicative of flowability and high packing density of the powder. 


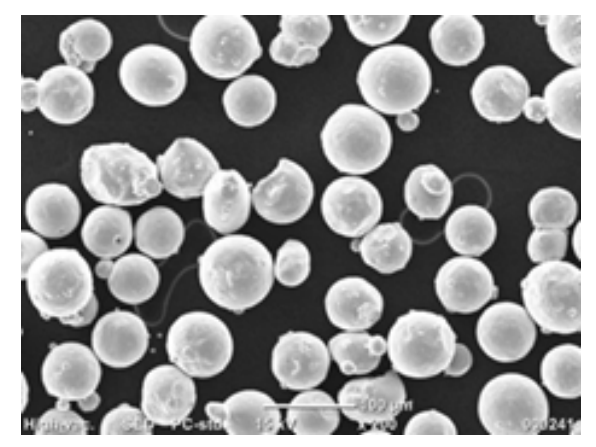

Figure 1 : SEM morphology of Ti-6Al-4V powder

In the present study, a LMD machine of the laboratory was used. It is equipped with IREPA LMD nozzles and a $2 \mathrm{~kW}$ laser. At first, process parameters selection has been made by focusing on porosity and lack of fusion defects. Then, Ti-6Al-4V blocks of $160 \times 90 \times 14 \mathrm{~mm} 3$ were fabricated on commercially titanium alloy using 1.1 to $1.6 \mathrm{KW}$ laser power, $2 \mathrm{~m} / \mathrm{min}$ feedrate and $8.7 \mathrm{~g} / \mathrm{min}$ powder flow rate. A $45^{\circ}$ Zig-zag alternative hatching strategy was used. The processing atmosphere was controlled to keep less than $50 \mathrm{ppm}$ of Oxygen. After the fabrication, the samples were stress relieved at $594^{\circ} \mathrm{C}$ for 2 hours and then cooled in environmental conditions. For tensile, LCF and crack propagation studies, the specimen extraction strategy is shown in Figure 2.

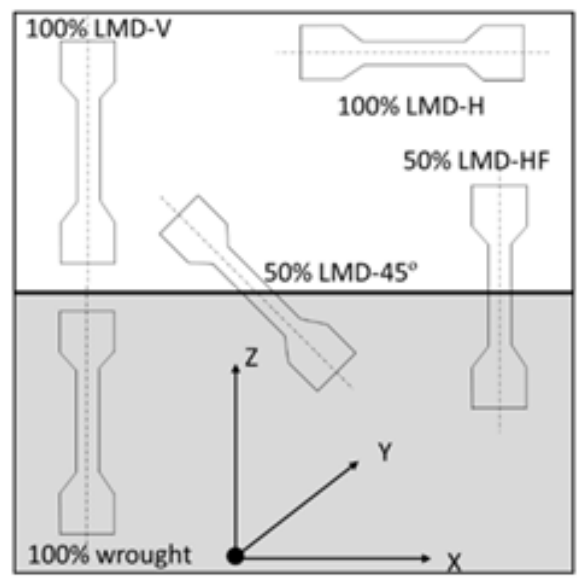

Figure 2: Specimen extraction strategy

Tensile testing

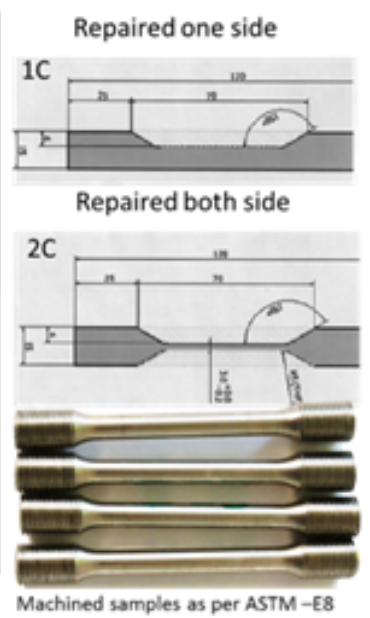


Tensile tests were performed on an INSTRON 5565 Universal Testing Machine at room temperature according to ASTM E8 with a test velocity of $2 \mathrm{~mm} / \mathrm{min}$. The tensile samples were sectioned at different places of the fabricated blocks, no interface porosity was observed in the fabricated samples. Four specimens of each condition and the average values were taken for consideration. As shown on Figure 3 and Figure 4, the standard deviation is very small for each type of sample and these results can be considered as repeatable.

As can be seen from Figure 3, the yield strength and ultimate tensile strengths are significantly equal to the wrought alloy. The repaired samples yield strength and UTS are greater than the wrought alloy. In contrast with the other measures (Figure 4), elongation results show differences between the AM samples types. For as built samples, the values are $20-25 \%$ below the reference material. For interface samples the gap is very large (over $75 \%)$ This could be explained by the grain orientation and size which affects ductility, as melting and solidification rates are faster in LMD compared to conventional manufacturing process. However, the ductility of LMDed components is almost independent of the direction of deposition.

The percentage of elongation of $\mathrm{H}$ and $\mathrm{V}$ samples are comparable to wrought alloy but the $45^{\circ}$, $\mathrm{HF}$ and repaired (1C and 2C) samples have less ductility.

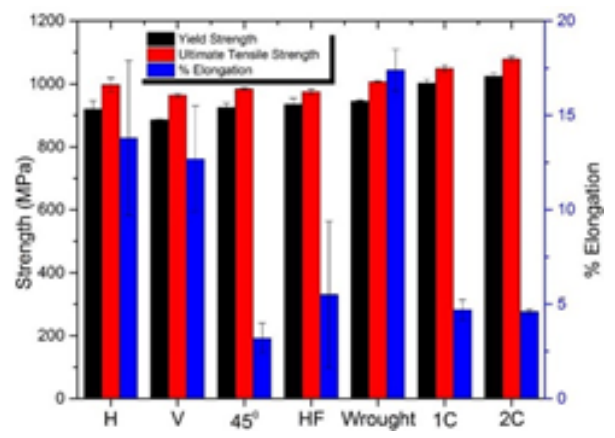

Figure 3 : Yield Strength, Ultimate tensile strength (UTS) and \% elongation for specimens with differences in location and orientation

According to the results obtained (Figure 3), the mechanical properties of the powder deposited specimen are comparable but little below the reference material properties. The Young Modulus for the powder deposited samples are very close, whatever the extracted sample. Their value is $6-7 \%$ below the reference sample. Reason being, that elastic property is almost similar to wrought materials. Also, compared to published literature, the obtained values are very good and can be explained by a good fusion process that did not generate harmful porosities in the fabricated samples. 


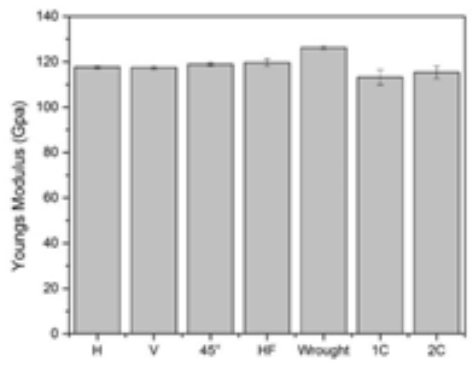

Figure 4 : Young's modulus for specimens with differences in location and orientation

\section{Low Cycle Fatigue (LCF) tests}

The same manufacturing parameters were employed to produce Low Cycle Fatigue samples. 76 samples were manufactured in total using the same extraction strategy than for tensile: $100 \%$ Horizontal (15), 100\%Vertical (15), $50 \% \mathrm{HF}(18)$ and $50 \%$ at $45^{\circ}(18)$ and $100 \%$ wrought (10). The specimen geometry was chosen according to ASTM E466.with a $6 \mathrm{~mm}$ diameter in the central zone. LCF tests were done on an INSTRON 2003 machine according to NF EN 6072 standard, at room temperature. Load levels were chosen in order to draw the StressCycle curves between $2.10^{4}$ and $1.10^{6}$ cycles. If the specimen has not broken at this time, the tests were stopped at $2.10^{6}$ cycles. The results are given in Figure 5:

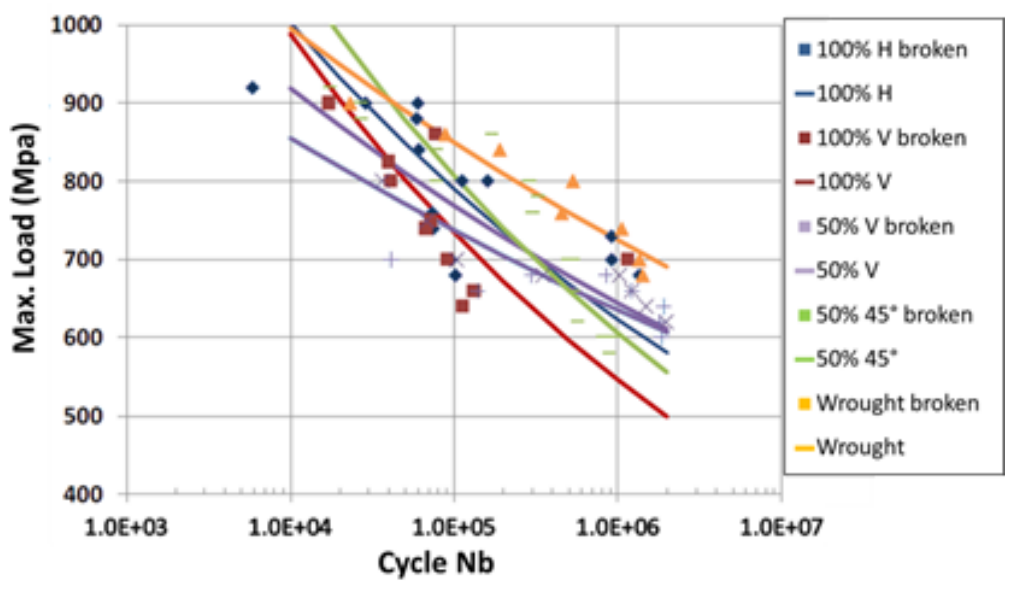

Figure 5: LCF results

The highlight the very good performance obtained with Additive Manufacturing. The repeatability is very good and the S-N curves are very similar. From a global point of view, wrought samples provide the best results whereas the S-N curve for the $100 \%$ as built samples is the lowest. The sample extraction direction does not have any significant impact, which is very interesting within the context of Ti-6Al-4Vcomponent repair. 
In addition, SEM observations of the fracture surfaces show a very sound material structure with very little porosity which is very promising.

\section{Crack Propagation}

A Crack propagation experimental campaign was conducted as well on the same LMD manufactured components. 18 specimen were designed according to ASTM E647-00 standard and the extraction strategy lead to test: $100 \%$ Horizontal (4), 100\% Vertical (4), 50\% Horizontal (3), 50\% Vertical (4) and 100\% Wrought (3). The test machine was a servo-hydraulic INSTRO 8850 with a FRACTOMAT system to measure the crack growth. All tests were conducted at frequencies between 20 and $30 \mathrm{~Hz}$ with a load ratio $\mathrm{Fmin} / \mathrm{Fmax}=0.1$, with the objective to determine the "Paris" law parameters and the crack propagation level.

The results for all 18 specimens are given in Figure 6, and the following conclusions can be made: the lowest crack propagation level is obtained with the $50 \%$ vertical samples (interface) while the highest level is obtained with the wrought samples. There is a very good repeatability among the samples of the same batch. The manufacturing direction (vertical samples vs horizontal samples) does not have a significant impact on the curves. However, concerning the propagation angle, it has to be noted that its stays at $0^{\circ}$ for all batches except for the $50 \%$ vertical where it goes around $8^{\circ}$ (in the LMD portion). As a result, the interface LMD/ substrate has an effect on the crack propagation direction.

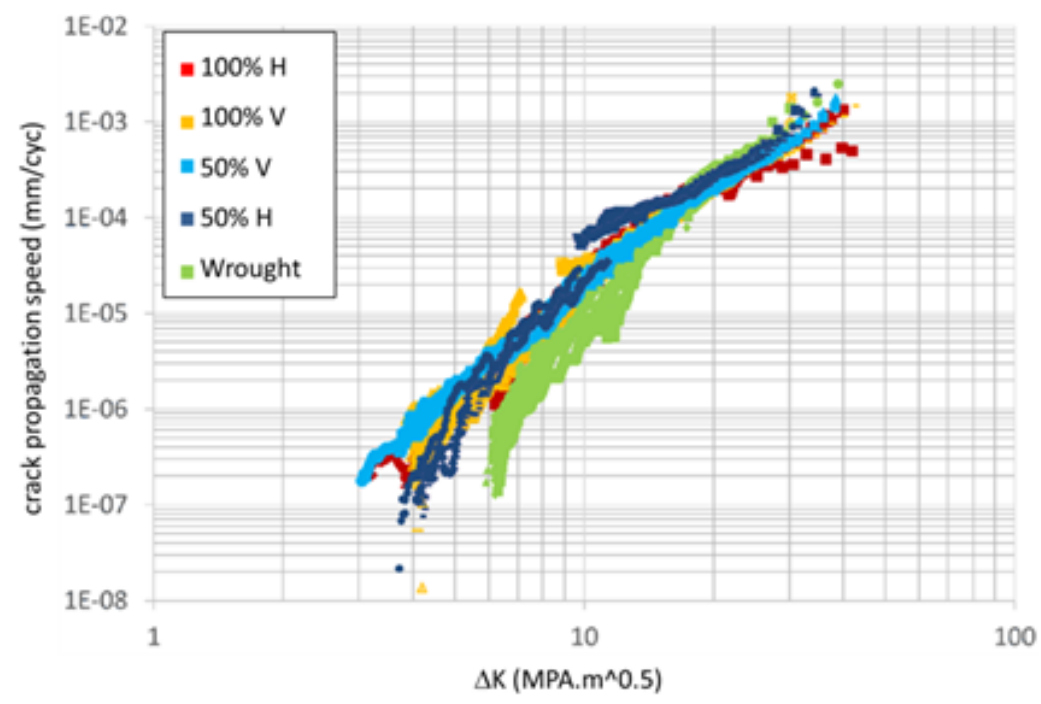

Figure 6: Crack propagation results

Discussion 
This experimental campaign focused on tensile, fatigue and crack propagation characteristics. The mechanical properties of the powder deposited specimen are comparable but below the reference material properties. The standard deviation is very small for each type of samples. These results can be considered as repeatable, which is profitable within the objective to propose automatic repair methods as well as Additive Manufacturing of complete Ti-6Al-4V components.

The relevance of the LMD process to produce sound repaired material is consequently validated. This manufacturing process can be considered as a valid solution to repair TI-6Al-4V aeronautical components. The next section of the paper introduces a semi-automatic method to repair this kind of components by using the LMD process with the selected parameters.

\section{$\underline{\text { Semi automatic repair method of Ti-6Al-4V components }}$}

A major difficulty with repair compared to production is the variety of configurations and it is necessary to adapt the process to this variety. This explains why most of the repairs are still done manually and rely on user know-how. To fill the gap, a semi-automatic method has been proposed by the research group [13]: it applies to external or internal defects that have been machined into a surface cavity. This cavity is to be refilled in such a way as to recover the local geometry of the part, without the need of a nominal CAD model and with minimal user intervention.

As given in Figure 7, the method can be segmented into several steps: part inspection and defect identification, machining of the defect into a cavity, cavity identification, Additive Manufacturing tool paths generation and cavity refill.

\section{Part inspection and defect identification}

At first, the defective metallic part is scanned by imaging equipment, such as radiography or CT scans. The scan data is then inspected for defect identification, either manually by an operator or automatically by machine vision algorithms such as in the work of Mery [14].

\section{Machining of the defect into a cavity}

A machining operation is usually necessary to prepare the repair. It consists of removing the defective material, in case of casting for example, and/or to make the geometry suitable for repair, in case of the worn parts for example. This operation is usually done manually and generates a cavity of the component surface that is not defined nor located on the CAD model of the component (if available).

\section{Cavity identification}

In very few cases, the machining tool paths can be recycled into scan paths for refilling the cavity [15] and the repair can be done immediately. But, in most cases, the cavity edges and volume have to be identified and this 
is the purpose of this step of the method, called InterSAC. Two prerequisites are needed to use InterSAC. It is first assumed that the shape of the machined cavity can be analytically represented, which is usually the case as the cavity opening consists of simple motions of the cutting tool. Then it is assumed that the original local geometry of the part in the cavity area is smooth and regular enough to be represented as an analytic surface.

The cavity area is scanned with a non-contact range sensor to obtain a 3D digital representation of the cavity and its neighbouring surface. InterSAC method is robust enough to be applied to partial range data where the cavity area and/or the neighbouring surface are partially truncated. The user also selects a canonical surface which represents the geometry of the machined cavity, as well as a surface that best represents the locally shape of the neighbouring surface (sphere, cone, cylinder, paraboloid, ellipsoid, hyperboloid, torus, quadric, superquadric, conic, bivariate polynomial...). If the type of neighbouring surface is unknown, the user may choose a type of surface of sufficiently high degree, so that it may correctly approximate the actual neighbouring surface.

Then, the RANSAC-based algorithm proceeds to estimate model parameters estimation based on the range data: two identification steps are performed in a sequential manner. In the second identification step, the algorithm only samples data points that correspond to the outliers of the firstly identified model. This helps to reduce the number of data points available for randomized sampling, thereby reducing the theoretical number of random samples to be drawn.

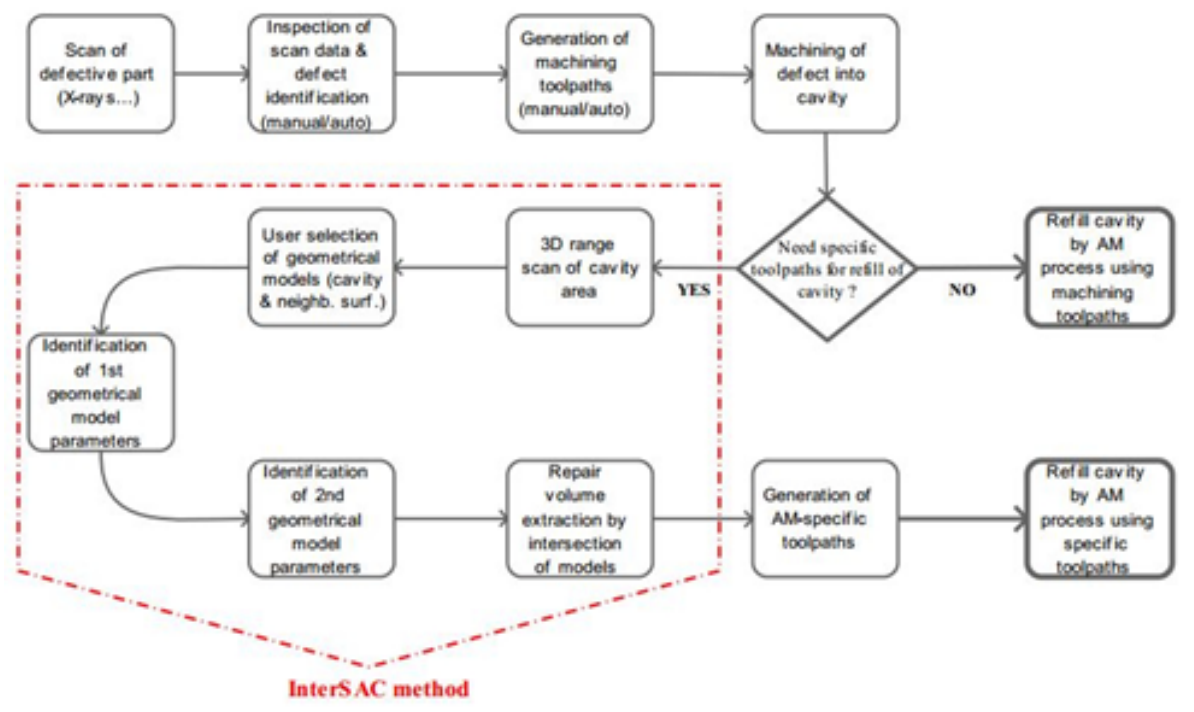

Figure 7: semi-automatic repair method

Once the parameters of the two geometrical models are estimated, an intersection operation generates the 3D repair volume. The 3D intersection curve defines the surface edge between the cavity and its neighbouring surface. Below the surface edge, the bottom of the 3D repair volume is defined by the geometrical model of the cavity bounded by the 3D intersection curve. Above the surface edge, the top of the repair volume is defined by 
the intersection surface, which corresponds to the neighbouring surface bounded by the 3D intersection curve. Hence, a 3D repair volume can be formed in such a way as to recover the local geometry of the metallic part in the repair area. An example of reconstruction is given in Figure 8 for the case of a slot cavity machined with ball end tool on a flat workpiece.

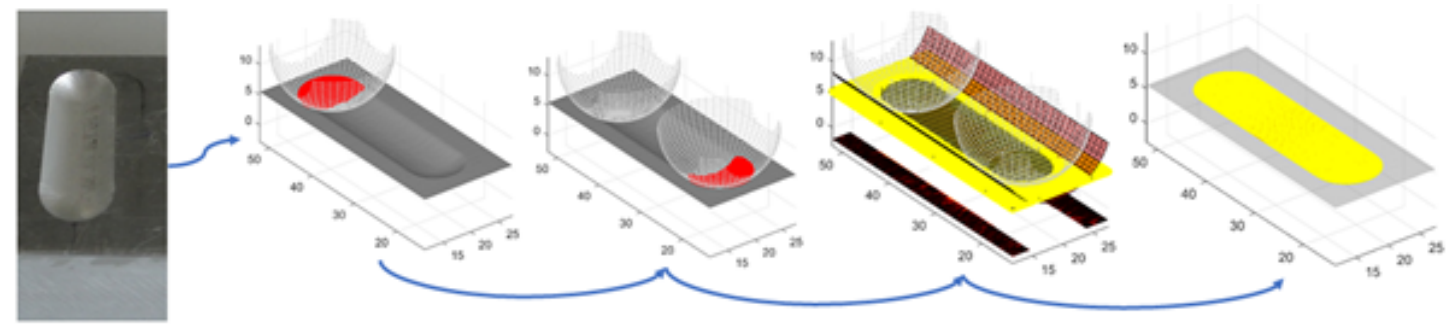

Figure 8: Repair volume extraction

At the end of this step, the repair volume is fully identified and localized on the part or the LMD machine workspace, and the repair LMD tool paths can be generated.

\section{Additive Manufacturing Tool Paths Generation and Cavity Refill}

By using the optimal parameters as defined in the previous section, the repair method generates the LMD scan paths and the associated machine code for Additive Manufacturing. If this latest operation is achieved successfully, the local shape of the metallic part is fully recovered, as shown in Figure 9.
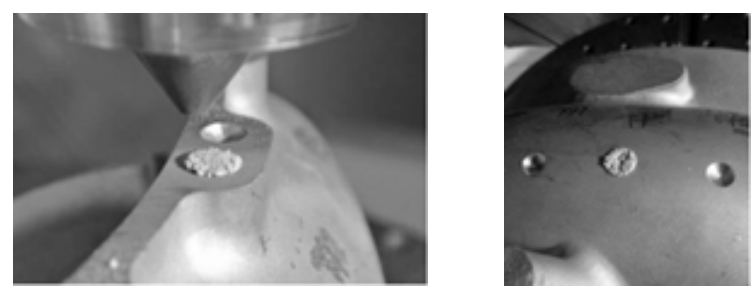

Figure 9: Examples of aeronautical components repair with the proposed method

\section{Finishing operations}

The last step of the method is to give the repair part its initial shape and surface properties by removing the excess material deposited. This operation is usually done by machining, using similar process and parameterization as the initial manufacturing of the component. 


\section{Conclusion}

This paper studied the capability of LMD process to be a valid solution for Ti-6Al-4V aeronautical components. The first section discussed the mechanical properties of LMD samples with a specific focus on the properties at the interfaces. The obtained results are very good not only in the perspective of repair but for manufacturing complete parts as well. Then, the second section introduced a semi automatic repair method which accuracy and performance do not rely on the availability of a CAD model. The combination of these two approaches increases the capability to repair high value aeronautical components by providing a reliable and repeatable method.

\section{$\underline{\text { Acknowledgements }}$}

Some works presented in this paper were done within the context of the H2020 European "Additive Manufacturing Optimisation and Simulation (AMOS)" project.

\section{$\underline{\text { References }}$}

[1] P. Muller, P. Mognol, and J.-Y. Hascoët, 'Modeling and control of a direct laser powder deposition process for Functionally Graded Materials (FGM) parts manufacturing', journal of materials processing technology, vol. 213, no. 5, pp. 685-692, 2013.

[2] D. Bourell et al., 'Materials for additive manufacturing', CIRP Annals, vol. 66, no. 2, pp. 659-681, Jan. 2017.

A. Saboori, D. Gallo, S. Biamino, P. Fino, and M. Lombardi, 'An Overview of Additive Manufacturing of Titanium Components by Directed Energy Deposition: Microstructure and Mechanical Properties', Applied Sciences, vol. 7, no. 9, p. 883, Sep. 2017.

[4] T. Wang, Y. Y. Zhu, S. Q. Zhang, H. B. Tang, and H. M. Wang, 'Grain morphology evolution behavior of titanium alloy components during laser melting deposition additive manufacturing', Journal of Alloys and Compounds, vol. 632, pp. 505-513, May 2015.

[5] J. Wang, L. Li, C. Tan, H. Liu, and P. Lin, 'Microstructure and tensile properties of TiCp/Ti6Al4V titanium matrix composites manufactured by laser melting deposition', Journal of Materials Processing Technology, vol. 252, pp. 524-536, Feb. 2018.

[6] J. Yu, M. Rombouts, G. Maes, and F. Motmans, 'Material Properties of Ti6Al4V Parts Produced by Laser Metal Deposition', Physics Procedia, vol. 39, pp. 416-424, Jan. 2012. 
[7] Y. Zhai, H. Galarraga, and D. A. Lados, 'Microstructure, static properties, and fatigue crack growth mechanisms in Ti-6Al-4V fabricated by additive manufacturing: LENS and EBM', Engineering Failure Analysis, vol. 69, pp. 3-14, Nov. 2016.

[8] Z. Zhao, J. Chen, H. Tan, X. Lin, and W. Huang, 'Evolution of plastic deformation and its effect on mechanical properties of laser additive repaired Ti64ELI titanium alloy', Optics \& Laser Technology, vol. 92, pp. 36-43, Jul. 2017.

[9] A. J. Pinkerton, W. Wang, and L. Li, 'Component repair using laser direct metal deposition', Proceedings of the Institution of Mechanical Engineers, Part B: Journal of Engineering Manufacture, vol. 222, no. 7, pp. 827-836, Jul. 2008.

[10] B. Graf, A. Gumenyuk, and M. Rethmeier, 'Laser Metal Deposition as Repair Technology for Stainless Steel and Titanium Alloys', Physics Procedia, vol. 39, pp. 376-381, Jan. 2012.

[11] C. Piya, J. M. Wilson, S. Murugappan, Y. Shin, and K. Ramani, 'Virtual Repair: Geometric Reconstruction for Remanufacturing Gas Turbine Blades', in Volume 9: 23rd International Conference on Design Theory and Methodology; 16th Design for Manufacturing and the Life Cycle Conference, Washington, DC, USA, 2011, pp. 895-904.

[12] J. Um, M. Rauch, J.-Y. Hascoët, and I. Stroud, 'STEP-NC compliant process planning of additive manufacturing: remanufacturing’, Int J Adv Manuf Technol, pp. 1-16, May 2016.

[13] J.-Y. Hascoët, S. Touzé, and M. Rauch, 'Automated identification of defect geometry for metallic part repair by an additive manufacturing process', Weld World, vol. 62, no. 2, pp. 229-241, Mar. 2018.

[14] D. Mery, 'Automated Radioscopic Inspection of Aluminium Die Castings', Materials Evaluation, vol. 65 , no. 6, pp. 643-647, 2006.

[15] I. Tabernero, A. Calleja, A. Lamikiz, and L. N. López De Lacalle, 'Optimal parameters for 5-axis Laser cladding', Procedia Engineering, vol. 63, pp. 45-52, 2013. 